\title{
Predicting Soybean Yield and Sudden Death Syndrome Development Using At-Planting Risk Factors
}

\author{
Mitchell G. Roth, ${ }^{1,2}$ Zachary A. Noel, ${ }^{1,3}$ Jie Wang, ${ }^{4}$ Fred Warner, ${ }^{1}$ Adam M. Byrne, ${ }^{1}$ and Martin I. Chilvers ${ }^{1,2,3, \dagger}$ \\ ${ }^{1}$ Department of Plant, Soil and Microbial Sciences, Michigan State University, East Lansing, MI 48824 \\ ${ }^{2}$ Genetics Program, Michigan State University, East Lansing, MI 48824 \\ ${ }^{3}$ Program in Ecology, Evolutionary Biology and Behavior, Michigan State University, East Lansing, MI 48824 \\ ${ }^{4}$ Department of Plant Biology, Michigan State University, East Lansing, MI 48824 \\ Accepted for publication 10 May 2019.
}

\begin{abstract}
In the United States, sudden death syndrome (SDS) of soybean is caused by the fungal pathogen Fusarium virguliforme and is responsible for important yield losses each year. Understanding the risk of SDS development and subsequent yield loss could provide growers with valuable information for management of this challenging disease. Current management strategies for $F$. virguliforme use partially resistant cultivars, fungicide seed treatments, and extended crop rotations with diverse crops. The aim of this study was to develop models to predict SDS severity and soybean yield loss using at-planting risk factors to integrate with current SDS management strategies. In 2014 and 2015, field studies were conducted in adjacent fields in Decatur, MI, which were intensively monitored for $F$. virguliforme and nematode quantities at-planting, plant health throughout the growing season, end-of-season SDS severity, and yield using an unbiased grid sampling scheme. In both years, $F$. virguliforme and soybean cyst nematode (SCN) quantities were unevenly distributed throughout the field. The distribution of $F$. virguliforme at-planting had a significant correlation with end-of-season SDS severity in 2015, and a significant correlation to yield
\end{abstract}

ABSTRACT in $2014(P<0.05)$. SCN distributions at-planting were significantly correlated with end-of-season SDS severity and yield in $2015(P<$ $0.05)$. Prediction models developed through multiple linear regression showed that $F$. virguliforme abundance $(P<0.001)$, SCN egg quantity $(P<0.001)$, and year $(P<0.01)$ explained the most variation in end-ofseason SDS $\left(R^{2}=0.32\right)$, whereas end-of-season SDS $(P<0.001)$ and end-of-season root dry weight $(P<0.001)$ explained the most variation in soybean yield $\left(R^{2}=0.53\right)$. Further, multivariate analyses support a synergistic relationship between $F$. virguliforme and $\mathrm{SCN}$, enhancing the severity of foliar SDS. These models indicate that it is possible to predict patches of SDS severity using at-planting risk factors. Verifying these models and incorporating additional data types may help improve SDS management and forecast soybean markets in response to SDS threats.

Keywords: disease control, pest management, soybean cyst nematode, soybean sudden death syndrome
Soybean (Glycine max [L.] Merr.) is one of the most cultivated and economically important crops in the United States but is prone to yield losses caused by many pathogens (Allen et al. 2017; Koenning and Wrather 2010). In North America, the fungal pathogen Fusarium virguliforme O'Donnell \& T. Aoki causes soybean sudden death syndrome (SDS). Fusarium virguliforme has no known sexual reproductive stage (Hughes et al. 2017) but can produce microconidia, macroconidia, and overwintering chlamydospores (Aoki et al. 2003). These spores can germinate into mycelia in the soil, which release cell wall-degrading enzymes after coming into contact with soybean roots (Chang et al. 2016) and develop an appressorium (Navi and Yang 2008) to facilitate infection. Soybean roots of any age can be infected (Gongora-Canul and Leandro 2011; Hartman et al. 2015). Initial symptoms develop as root discoloration and root rot, but eventually foliar symptoms develop as interveinal chlorosis, necrosis, and premature defoliation, leading to yield loss (Hartman et al. 2015).

\section{${ }^{\dagger}$ Corresponding author: M. I. Chilvers; chilvers@msu.edu}

Funding: This study was supported by the Michigan Soybean Promotion Committee, Bayer CropScience, the Michigan State University Plant Science Fellowship, the Everett "Tex" Beneke Fund, and the Syngenta Agricultural Scholarship.

*The $e$-Xtra logo stands for "electronic extra" and indicates that one supplementary figure and one supplementary table are published online.

The authors declare no conflict of interest.

This article is in the public domain and not copyrightable. It may be freely reprinted with customary crediting of the source. The American Phytopathological Society, 2019.
However, F. virguliforme remains in the root tissues throughout foliar disease development and has never been isolated from above-ground tissues (Roy et al. 1989). SDS commonly appears in heterogeneous patches within a field and differentially affects yield throughout a single field (Hartman et al. 2015). Evidence suggests that foliar SDS symptoms are the result of $F$. virguliforme penetration to the vascular tissues (Islam et al. 2017a, b; Navi and Yang 2008), where various secondary metabolites and protein toxins are secreted into the xylem and translocated to the foliar tissues (Abeysekara and Bhattacharyya 2014; Brar et al. 2011; Chang et al. 2016; Pudake et al. 2013).

Heterodera glycines Ichinohe, the soybean cyst nematode (SCN), is commonly found in fields throughout the United States and also causes significant yield loss in soybean (Allen et al. 2017; Koenning and Wrather 2010). Multiple plant-parasitic nematodes have been implicated in disease complexes with plant pathogenic fungi and oomycetes, including SCN with $F$. virguliforme in soybean (Back et al. 2002; Rupe 1989; Rupe et al. 1993). Independently, both $F$. virguliforme and SCN can penetrate to the vascular tissue, but it is unknown if the two pathogens interact before reaching the vascular tissues, or if $F$. virguliforme preferentially colonizes wounds caused by SCN or other nematodes. Previous studies have shown that $F$. virguliforme can colonize $\mathrm{SCN}$ eggs and cysts (Donald et al. 1993; McLean and Lawrence 1995). Field studies have found that foliar SDS severity is worse when soybeans are inoculated with both pathogens compared with $F$. virguliforme alone (Brzostowski et al. 2014; McLean and Lawrence 1993; Scherm et al. 1998; Westphal et al. 2014; Xing and Westphal 2013). Another recent study showed that managing SCN through partially resistant cultivars also reduced SDS severity (Kandel et al. 2017). Together, these studies indicate a unique relationship between $F$. virguliforme and SCN that 
leads to severe SDS, with evidence suggesting that the relationship is synergistic (Xing and Westphal 2013). Because SCN cannot cause SDS independently, the relationship is not additive and any relationship with $F$. virguliforme that enhances SDS severity could be classified as synergistic. However, other field studies have failed to find evidence to support a synergistic relationship leading to increased SDS (Hershman et al. 1990; Marburger et al. 2014; Rupe et al. 1993). A factorial greenhouse experiment revealed that these pathogens caused a more significant reduction in root mass together, compared with either pathogen alone, but there was no increase in foliar SDS because of the presence of SCN (Gao et al. 2006).

Current management strategies for SDS use partially resistant cultivars, seed treatments with fungicides (Kandel et al. 2018), crop rotation (Leandro et al. 2018), and soil tillage practices (Hartman et al. 2015); however, each strategy has drawbacks. Crop rotations may fail because $F$. virguliforme can colonize other crop and weed species without causing visual symptoms, allowing inoculum to build up (Kolander et al. 2012). Extended crop rotations with diverse crops can be costly depending on crop markets and is not widely adopted by growers. Soil tillage may fail because F. virguliforme can overwinter in soybean and corn residue (Navi and Yang 2016). Seed treatments containing the fungicide fluopyram (BASF, Ludwigshafen, Germany) have shown efficacy in vitro and in planta (Gaspar et al. 2017; Kandel et al. 2016; Sang et al. 2018; Wang et al. 2016). However, few other fungicides show significant reduction of $F$. virguliforme inside plant roots (Weems et al. 2015), and reliance on a single mode of action fungicide is undesirable because of its potential to select for fungicide resistance. Identification of quantitative trait loci (QTLs) has led to improved genetic resistance to SDS; however, resistance is quantitative and complex because separate QTLs control resistance to root and foliar symptoms (Chang et al. 2018; de Farias Neto et al. 2007; Kazi et al. 2008; Lightfoot 2015; Luckew et al. 2013; Tan et al. 2019; Wen et al. 2014). Data suggest that suppressing foliar SDS symptoms significantly improves yield (Luo et al. 2000) and some breeding efforts focus solely on foliar SDS symptoms (Wen et al. 2014). However, cultivars with no foliar symptoms may still be heavily colonized by $F$. virguliforme (Wang et al. 2019). The impact of root rot on yield is difficult to estimate, and the impact of root rot caused by $F$. virguliforme in soybean is currently unknown. In addition, some SDS resistance QTLs overlap with known SCN resistance QTLs, complicating the role of each in specific resistance to SDS or SCN (Meksem et al. 1999; Srour et al. 2012).

Another SDS management strategy that has yet to be widely implemented is risk prediction. Risk prediction has been used for other soybean diseases, such as Asian soybean rust and Sclerotinia stem rot (Del Ponte et al. 2006; Mila et al. 2004; Willbur et al. 2018a, b), as well as SDS at the regional level (Scherm and Yang 1996, 1998; Scherm and Yang 1999). These previous studies modeled SDS development using environmental data, such as soil temperature, soil water potential (Scherm and Yang 1996), cold stress response, and geographic location (Scherm and Yang 1999). Although environment plays a key role in disease development, the presence of the pathogen may not be evenly distributed across a field. These climate-driven regional models indicated a highly unfavorable environment for SDS development in Minnesota, South Dakota, and Nebraska (Scherm and Yang 1999) even though $F$. virguliforme has been subsequently identified there and is consistently ranked in the top five most damaging soybean diseases in the northern United States (Allen et al. 2017). Incorporating pathogen abundance data into SDS predictive models at the field level is essential for improved prediction because $F$. virguliforme is present and causing SDS in locations predicted to have an unfavorable environment for SDS.

In this study, we aimed to incorporate $\mathrm{SCN}$ and $F$. virguliforme abundance data into SDS predictive models at the field level. We used a high-intensity sampling method over 2 years to quantify SCN and $F$. virguliforme and examine their spatial distribution in a naturally infested field in Michigan. The goals of this study were to (i) determine the spatial distribution of $F$. virguliforme and SCN in field sites naturally infested with both pathogens, (ii) identify at-planting risk factors for modeling SDS severity and yield, and (iii) explore synergistic effects of $F$. virguliforme and nematodes on SDS severity.

\section{MATERIALS AND METHODS}

Field and sampling design. Two adjacent field sites in Decatur, MI, with sandy loam soils were selected for field trials in 2014 and 2015. Both fields are naturally infested with $F$. virguliforme and $\mathrm{SCN}$ and managed under a corn-soybean rotation with minimal-till conditions using a chisel plow. In both years, the field was the same size, measuring $9.1 \mathrm{~m} \times 48.8 \mathrm{~m}$. The fields were divided into 24 plots, each measuring $3.0 \mathrm{~m} \times 6.1 \mathrm{~m}$. Each plot contained four rows of soybean with $0.8-\mathrm{m}$ row spacing, planted at a density of approximately nine seeds per $0.3 \mathrm{~m}$. In 2014, untreated Asgrow AG2534 seeds were planted, and in 2015, Asgrow AG2535 seeds (Monsanto, St. Louis, MO) containing the company's "complete" Acceleron treatment package (Rossman et al. 2018) were planted. These seed choices represent common varieties and treatments available to Michigan growers. Both varieties are rated 7 of 9 for SDS tolerance by the seed company, where $1=$ excellent and $9=$ poor. Soybeans were planted in early May of each year. Fields received natural rainfall with additional overhead irrigation, resulting in an average weekly water volume of $2.7 \mathrm{~cm}$ in 2014 and $3.0 \mathrm{~cm}$ in 2015 . The plots were harvested with an Almaco combine equipped with a $1.5-\mathrm{m}$ head, and yield was determined with the HarvestMaster system (Juniper Systems, Logan, UT). In 2014, the center two rows of each four-row plot were harvested, providing 24 data points, whereas in 2015 all four rows were harvested, two rows at a time, providing 48 data points for yield.

A predetermined grid sampling scheme was used so that sampling locations were in the outside rows of the 24 plots, $1.5 \mathrm{~m}$ from the end of the row, for a total of 96 sampling areas. Sampling locations were marked with a stake to ensure future samples could be obtained from the same location throughout the season. Soil samples were collected in-furrow, within 3 days of planting and again after harvest. Soil was collected using a $2.5-\mathrm{cm}$ diameter, 15.2-cm deep soil core. Six soil cores were collected per sampling location and homogenized in a plastic bag. Three soybean plants were collected at each sampling location at an early vegetative growth stage (V3) and a late reproductive growth (R5) stage (Fehr et al. 1971).

F. virguliforme and nematode detection. Root samples were separated from foliar tissues, oven-dried at $50^{\circ} \mathrm{C}$ for $48 \mathrm{~h}$, and ground using a Christy Mill (Christy Turner Ltd, Ipswich, Suffolk, U.K.). DNA was extracted from $100 \mathrm{mg}$ of ground root tissue using an automated phenol-chloroform technique performed on the AutoGen 850 Alpha system (AutoGen Inc, Holliston, MA), or the same phenol-chloroform protocol performed manually. DNA extractions from soil were performed in triplicate using $500 \mathrm{mg}$ ofhomogenized soil with the FastDNA SPIN Kit for Soil (MP Biomedicals, Santa Ana, CA), and the DNA was quantified using a Nanodrop 1000 (Thermo Fisher Scientific, Waltham, MA). Two microliters of DNA, at concentrations ranging from 30 to 110 $\mathrm{ng} / \mu \mathrm{l}$, were used in qPCR reactions to determine the level of $F$. virguliforme using the primers, probes, and cycling conditions reported by Wang et al. (2015). The total DNA concentration of each extraction was used to normalize the results obtained by qPCR into a ratio of femtograms of $F$. virguliforme DNA detected per nanogram of total DNA. Two technical replicates of qPCR were performed on each DNA extract in a StepOnePlus thermal cycler (v2.3; Applied Biosystems, Foster City, CA). Quantifying $F$. virguliforme from root samples was performed by taking the 
$\mathrm{Ct}_{\text {soy }}-\mathrm{Ct}_{\mathrm{Fv}}$, where $\mathrm{Ct}_{\text {soy }}$ represents the cycle threshold for amplification of the soybean $\beta$-tubulin reference gene (Wang et al. 2019) and $\mathrm{Ct}_{\mathrm{Fv}}$ represents the cycle threshold for amplification of the $F$. virguliforme qPCR target. In this way, samples with high levels of $F$. virguliforme (low $\mathrm{Ct}$ ) have a high calculated value, which is intuitively interpreted as high levels of $F$. virguliforme.

Soil samples collected at-planting and after harvest also were used for classifying and quantifying SCN, spiral nematodes (Heliocotylenchus spp.), lesion nematodes (Pratylenchus spp.), and dagger nematodes (Xiphinema spp.), based on morphology. One hundred milliliters of homogenized soil per sampling location was submitted to the Michigan State University Diagnostics Services (https://pestid.msu.edu/) for identification and quantification. Nematodes were isolated from soil using a modified soil centrifugation and floatation procedure (Jenkins 1964) and quantified under a dissecting microscope in a counting dish.

Tracking SDS development. Three plant samples were collected at each sampling point at growth stages V3 and R5, placed in a cooler, and transported back to Michigan State University. Foliar SDS incidence was calculated on each individual plant as the percentage of leaves showing foliar symptoms, and severity was assessed on symptomatic plants using a scale from 0 to 9 , with increments of 0.5 , that was developed at Southern Illinois University (J. Bond, unpublished data). Ratings of $0=$ no visible symptoms, $1=1$ to $10 \%$ of leaf surface chlorotic and/or 1 to $5 \%$ necrotic, $2=10$ to $20 \%$ chlorotic and/or 6 to $10 \%$ necrotic, $3=20$ to $40 \%$ chlorotic and/or 11 to $20 \%$ necrotic, $4=40$ to $60 \%$ chlorotic and/or 21 to $40 \%$ necrotic, $5=>60 \%$ chlorotic and $/$ or $>40 \%$ necrotic, 6 = premature leaf drop up to one-third defoliation, $7=$ premature leaf drop up to two-thirds defoliation, $8=$ premature leaf drop greater than two-thirds defoliation, $9=$ premature plant death (Supplementary Fig. S1). The 0.5 increment was applied when symptoms exceeded the requirements of one integer but did not meet the full requirements of the next integer, or when averaging symptomology across plants. The SDS severity and incidence measurements were converted to a normalized disease index (DX) score on a scale from 0 to 100 by multiplying incidence times severity, then dividing by the maximum severity score of 9 (Njiti et al. 2001). The roots of the sampled plants were washed with water and rated for discoloration and root decay, on a percentage scale from 0 to 100. The mean of the three plants was used to represent each sampling location and used for subsequent correlation analyses. These roots were dried as described previously, and dry weight was collected. Roots were then processed for DNA extraction and qPCR analysis as described previously.

Foliar SDS incidence and severity measurements were also collected in the field at growth stages R5 (Fehr et al. 1971). Foliar SDS incidence was observed as the percentage of plants in a given region showing foliar SDS, and severity was rated using the 0 to 9 scale detailed previously. These incidence and severity ratings were also transformed into a DX score, and the R5 SDS DX represents the end-of-season SDS severity in all analyses. In 2014, SDS incidence and severity were collected on a plot-wide basis, providing 24 data points. However, significant variation was observed within each plot in 2014, where some areas of severe SDS were averaged with areas of low SDS, which decreased spatial resolution of SDS in the field. Therefore, in 2015, foliar SDS severity and incidence ratings were measured only on approximately 25 plants that were in the same row as, and within $1 \mathrm{~m}$ of, each sampling location. This provided 96 data points, decreased within-plot variability, and increased spatial resolution of SDS in the field.

Data analysis. The 2014 and 2015 data sets were analyzed separately for Lloyd's index of patchiness (LIP), spatial interpolation, and Pearson's correlations. The 2014 and 2015 data were combined before 3-dimensional (3D) modeling, principal coordinates analysis (PCoA), and two-way stepwise multiple linear regression. The mean values of all variables collected for both years are also provided (Supplementary Table S1). All data were analyzed in $\mathrm{R}$ version 3.2.0 (R Core Team 2015, Vienna, Austria). Data and R scripts for these analyses can be found online at https://github.com/ rothmi12/SDS-Risk-Assessment.

The qPCR data obtained from soil samples were normalized to their respective total sample DNA concentration obtained to account for differences in DNA extraction efficiency per soil sample. This ratio represents the femtograms of $F$. virguliforme DNA detected per nanogram of total DNA extracted. The ratio of F. virguliforme DNA was used in all interpolation, correlation, linear regression, and pathway analyses. However, for LIP and PCoA, raw DNA quantities from qPCR (in femtograms) from soil samples were used to meet the assumption of count data for these analyses. Using density or proportional data instead of count data can cause incorrect calculations in these analyses (Bez 2000).

Contour plots were developed by using the "Krig" and "surface" functions within the "fields" R package (Nychka et al. 2017). Briefly, each data point for each variable was assigned an X and $\mathrm{Y}$ coordinate (in meters) based on their location within the field. A stationary covariance model was applied to the data to generate predictions of values from all areas, sampled and not sampled. The predicted data significantly correlated with the observed values in all cases (adjusted $R^{2}>0.86, P<0.001$ ), suggesting that the kriging models accurately display values in nonsampled regions.

Pearson's correlation analyses were done using the "stats" R package (R Core Team). Briefly, all variables were combined into a single matrix, where each row represented the same sampling area described previously. Then, each variable was independently correlated to all other variables and assigned a $P$ value and plotted for visualization of all pairwise correlations. $P$ values were adjusted using a false discovery rate correction with the "p.adjust" function from the "stats" package to reduce the chance of obtaining falsepositive significant correlations associated with multiple testing (Noble 2009). Only correlations between risk factors and end-ofseason SDS or yield are presented (Table 1).

A 3D scatter plot was made using the "plot3D" R package (Soetaert 2017). A PCoA was performed using only the at-planting $F$. virguliforme quantity (femtograms per nanogram of extracted DNA) and at-planting nematode quantities to investigate potential synergism in the development of SDS. PCoA was performed by calculating a dissimilarity matrix of Bray-Curtis distances using the “vegan" R package (Oksanen et al. 2018), which is commonly used for analyzing species abundance data (Bray and Curtis 1957; Faith et al. 1987). The "cmdscale" function of the "stats" $R$ package was then used to run the PCoA. Principal coordinate scores of the first two dimensions, which correspond to the dimensions with the highest variance, were plotted using the R package "ggbiplot" (Vu 2011), and points were colored by the R5 SDS DX.

Two-way stepwise multiple linear regression was implemented to develop models of best fit using the "MASS" R package (Venables and Ripley 2002), with cross-validation performed using the "caret" R package (Kuhn 2018). All models initially included all variables collected before the response variable so that each model had predictive capacity. For example, variables used in the initial model to predict R5 SDS included at-planting risk factors and V3 plant variables, but not R5 plant variables. All models were assessed using the Bayesian information criterion (BIC), which penalizes models for the number of factors included in the model to prevent overfitting the observed data. Each variable was dropped from each model and reassessed via BIC. If the model was not significantly worse, the variable was left out of the model, another variable was dropped, and the model was reassessed via BIC. If the model was significantly worse without the variable, the variable was placed back into the model, a different variable was dropped, and the model was reassessed via BIC. This iterative process was repeated until the best model was identified and no more variables could be dropped without significantly affecting the model. Finally, each of the best models was trained with a randomly selected subset of the data, representing $75 \%$ of the data. The models were subjected to a 
10-fold cross-validation, then used to make predictions on the remaining $25 \%$ of the data. The training and prediction were repeated 1,000 times, randomly selecting the training and testing data each time. After each training, the predicted values were correlated to the observed data, and an $R^{2}$ value was obtained for the cross-validation. Model accuracy was determined by the $R^{2}$ of the cross validations, also called $\mathrm{Q}^{2}$ (Alexander et al. 2015). The best models were formatted into a confirmatory factor analysis model for pathway analysis with the "lavaan" (Rosseel 2012) and "semPlot" packages (Epskamp 2017) in R. The pathway analysis was recreated by hand using Cytoscape v3.7.1 (Shannon et al. 2003).

\section{RESULTS}

Distribution of $F$. virguliforme and $H$. glycines in the soil. The field trials were in two different but adjacent fields in 2014 and 2015. Patches of severe SDS and yield loss were seen in the fields in both years. Many of the soil risk factors had an LIP $>1$ (Table 1), indicating that the variance of these variables exceeds the mean, which can be interpreted as significant aggregation (Bez 2000; Lloyd 1967). Subsequently, the physical locations of this aggregation were determined via kriging. In 2014 and 2015, areas of elevated at-planting $F$. virguliforme and SCN eggs visually overlapped with areas that developed more severe SDS and had reduced soybean yield (Fig. 1).

Pearson's correlations to end-of-season SDS DX and yield. Pearson's correlation analysis was used to determine if the at-planting and midseason variables were significantly correlated to end-of-season SDS and yield (Table 1). Nine variables had a significant correlation to end-of-season (R5) SDS DX or yield in at least one year $(P<0.05$, Table 1$)$. However, only root dry weight at R5 and SDS DX of collected R5 plants had a significant correlation to R5 SDS DX in both years. Similarly, only SDS DX of collected R5 plants had a significant correlation to yield in both years. Yield and R5 SDS DX had a significant negative correlation in both years $(P<0.05$, Table 1$)$.
The at-planting $F$. virguliforme quantities positively correlated with end-of-season SDS DX in both years, although it was statistically significant only in $2015(P<0.05$, Table 1$)$. In addition, at-planting $F$. virguliforme quantities had a negative correlation with yield in both years, although it was statistically significant only in $2014(P<0.05$, Table 1$)$. At-planting SCN cysts, eggs, and juveniles all had positive correlations with end-of-season SDS DX and negative correlations with yield in both years, but were significant only in 2015. Midseason plant variables, such as V3 root rot and R5 root dry weight, had some significant correlations to end-of-season SDS DX and yield, as expected (Table 1).

Modeling yield loss and SDS severity. The best model to predict soybean yield, determined by two-way stepwise multiple linear regression and assessment via the BIC, consisted of only two factors: R5 SDS DX and R5 root dry weight (Table 2). The best model to predict R5 SDS DX consisted of three factors: year, atplanting $F$. virguliforme quantity, and at-planting SCN eggs (Table 2). As R5 root dry weight was a significant factor in the yield model, we further determined that it is significantly impacted by two factors: V3 root rot and at-planting SCN cysts (Table 2). For each of these three models, interaction terms between the explanatory variables were added to the model and reassessed. Two of the models had marginally improved $R^{2}$ values, but none of them had improved $P$ values (data not shown). In addition, multicollinearity of independent factors within the final models was examined using variance inflation factors (VIFs), and all were $<2.5$. Because multicollinearity does not inhibit interpretation of model predictions and VIF values were $<10$, multicollinearity was not regarded as a significant concern (Kutner et al. 2005; O'Brien 2007). The $R^{2}$ values of the model cross validations $\left(Q^{2}\right)$ was lower than the original model $R^{2}$ value, with the exception of the R5 root dry weight model (Table 2). These models were visualized in a pathway analysis, which shows the relative contribution of each risk factor on each dependent variable, along with covariant relationships with other risk factors (Fig. 2). Pathway analysis showed that the risk factors included in the yield, R5 SDS DX, and R5 root dry weight models have positive covariance, particularly between

TABLE 1. Lloyd's index of patchiness (LIP) and correlation coefficients $(r)$ to end-of-season sudden death syndrome (SDS) disease index (DX) and yield for variables collected throughout the growing season

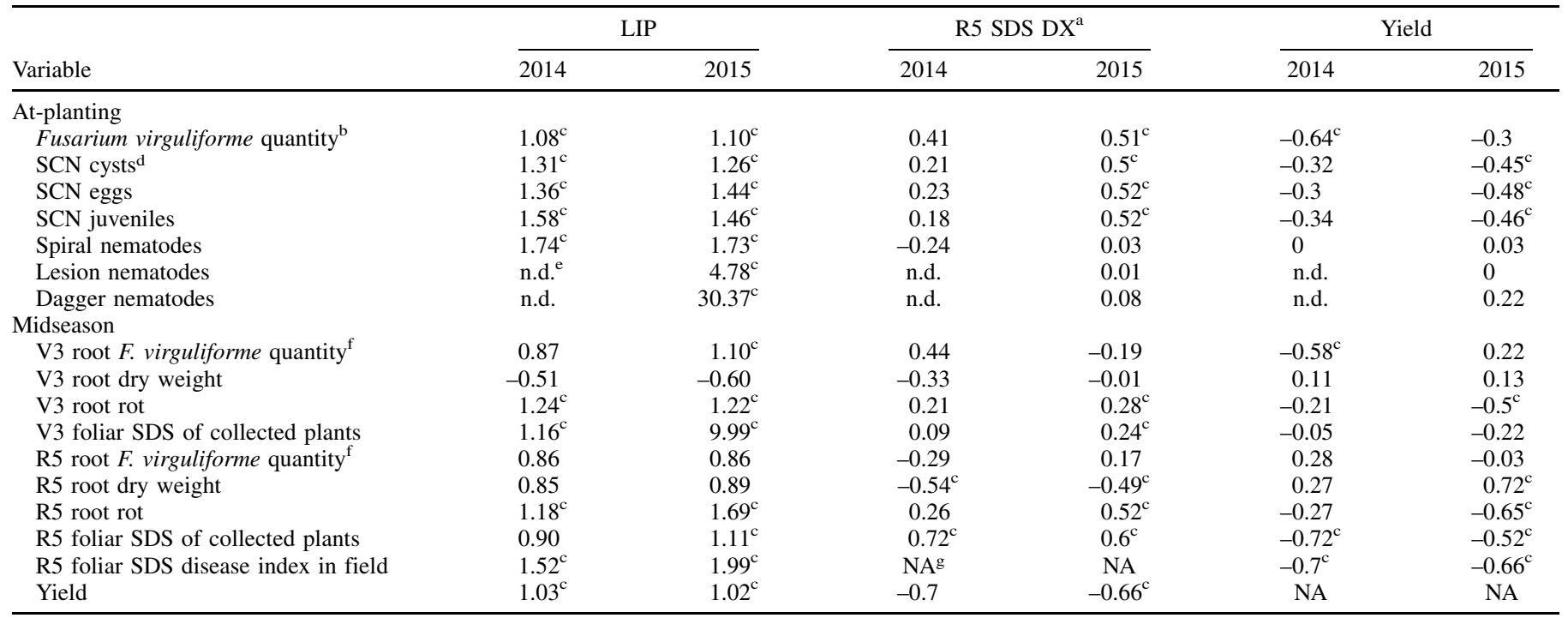

${ }^{a}$ R5 = soybean reproductive growth stage R5.

${ }^{\mathrm{b}}$ For LIP, femtograms of $F$. virguliforme DNA detected. For correlations with R5 SDS DX and yield, femtograms of $F$. virguliforme DNA per nanogram of total DNA extracted.

${ }^{c}$ LIP values are $>1$, indicating significant aggregation; $r$ values with false discovery rate-corrected $P<0.05$.

${ }^{\mathrm{d}} \mathrm{SCN}=$ soybean cyst nematode.

e n.d. = not detected in that year.

${ }^{\mathrm{f}}$ Calculated as $\mathrm{Ct}_{\mathrm{soy}}-\mathrm{Ct}_{\mathrm{Fv}}$, as described in the materials and methods section; V3 = soybean vegetative growth stage $\mathrm{V} 3$.

g NA = not applicable. 
at-planting $F$. virguliforme and SCN egg and cyst quantities, which together increase R5 SDS DX and decrease R5 root dry weight, which both affect yield (Fig. 2).

Multivariate analysis of $F$. virguliforme and nematode distributions. There was a positive correlation between atplanting $F$. virguliforme and at-planting SCN quantities in both years, but this was significant only in 2015 (2014: cysts $r=0.38$, eggs $r=0.29$, juveniles $r=0.30 ; 2015$ : cysts $r=0.38$, eggs $r=0.39$, juveniles $r=0.38$, all $P<0.001$ ). Using a 3D plot of at-planting $F$. virguliforme, at-planting SCN juveniles, and end-of-season SDS
DX, we found that at low, nonzero $F$. virguliforme levels, the severity of SDS increases as SCN increases (Fig. 3A). Similarly, with high at-planting $F$. virguliforme quantities, the severity of SDS also increases as the number of SCN eggs increases (Fig. 3A). Using PCoA, we investigated additional nematode species and found that the first two dimensions explained 54.0 and $45.2 \%$ of the variation, respectively (Fig. 3B). Both at-planting $F$. virguliforme quantities and at-planting SCN juveniles had significant correlations with these dimensions ( $\mathrm{x}$ and $\mathrm{y}$ axes $)(P<0.001)$, and the impact of year is also apparent. Although both at-planting $F$. virguliforme quantity

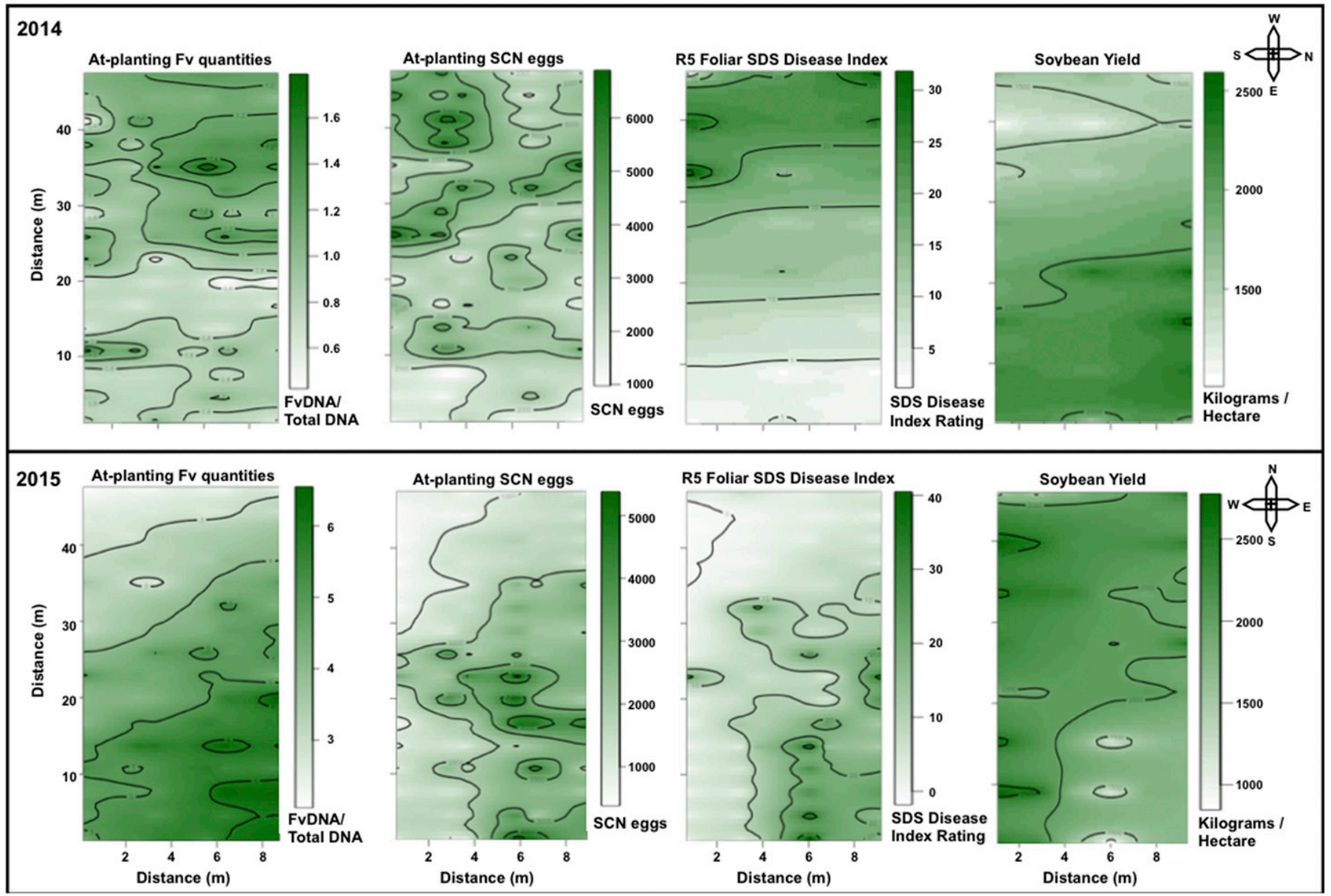

Fig. 1. Contour plots determined via kriging of the grid-sampled data representing Fusarium virguliforme (Fv) DNA quantities, soybean cyst nematode (SCN) egg quantities, soybean reproductive growth stage R5 foliar sudden death syndrome (SDS) disease index ratings, and soybean yield in 2014 (top) and 2015 (bottom). F. virguliforme quantities are shown as a proportion of $F$. virguliforme DNA detected (femtograms) from total DNA extracted (nanograms). SCN eggs are shown as the quantity of eggs detected per $100 \mathrm{~cm}^{3}$ of soil. R5 foliar SDS disease index is determined on a scale from 0 to 100. Yield is shown in kilograms per hectare. In all plots, white represents low values, and green represents high values.

TABLE 2. Prediction models and cross validations for yield, soybean sudden death syndrome (SDS) disease index (DX), and root dry weight models

\begin{tabular}{|c|c|c|c|c|c|c|c|c|}
\hline Response variable & $\begin{array}{l}\text { Explanatory } \\
\text { variables }^{\mathrm{a}}\end{array}$ & $\begin{array}{l}\text { Parameter } \\
\text { estimate }\end{array}$ & $\begin{array}{c}\text { Parameter } \\
\text { standard error }\end{array}$ & $P$ & Adjusted $R^{2}$ & Overall $P$ & $\begin{array}{c}\text { Average } \mathrm{R} \text { of predicted } \\
\text { versus actual }\end{array}$ & $\mathrm{Q}^{2^{\mathrm{b}}}$ \\
\hline \multirow[t]{2}{*}{ Yield } & R5 SDS DX & -0.351 & 0.563 & $<0.001$ & \multirow[t]{2}{*}{0.5310} & \multirow[t]{2}{*}{$<0.001$} & \multirow[t]{2}{*}{0.5740} & \multirow[t]{2}{*}{0.349} \\
\hline & R5 root dry weight ${ }^{\mathrm{c}}$ & 1.951 & 0.068 & $<0.001$ & & & & \\
\hline \multirow[t]{3}{*}{ R5 SDS disease index } & Year & -9.460 & 3.085 & 0.003 & \multirow[t]{3}{*}{0.3162} & \multirow[t]{3}{*}{$<0.001$} & \multirow[t]{3}{*}{0.4607} & \multirow[t]{3}{*}{0.224} \\
\hline & At-planting $\mathrm{Fv}$ qPCR ${ }^{\mathrm{d}}$ & 2.506 & 5.935 & $<0.001$ & & & & \\
\hline & At-planting SCN eggs & 0.003 & 0.001 & $<0.001$ & & & & \\
\hline \multirow[t]{2}{*}{$\mathrm{R} 5$ root dry weight ${ }^{\mathrm{c}}$} & $\mathrm{V} 3 \operatorname{root}^{\operatorname{rot}^{\mathrm{e}}}$ & -0.029 & 0.009 & 0.005 & \multirow[t]{2}{*}{0.2300} & \multirow[t]{2}{*}{$<0.001$} & \multirow[t]{2}{*}{0.5175} & \multirow[t]{2}{*}{0.27} \\
\hline & At-planting SCN cysts & -0.026 & 0.009 & 0.001 & & & & \\
\hline
\end{tabular}

a $\mathrm{R} 5=$ soybean reproductive growth stage $\mathrm{R} 5, \mathrm{FV}=$ Fusarium virguliforme, $\mathrm{SCN}=$ soybean cyst nematode, $\mathrm{V} 3=$ soybean vegetative growth stage $\mathrm{V} 3$.

b $\mathrm{Q}^{2}$ represents the average $R^{2}$ statistic of 1000 replications of a 10 -fold cross validation of each model.

c Dry weight in grams.

d Femtograms of $F$. virguliforme DNA per nanogram of total DNA extracted.

e In percentage of root system rotten. 
and at-planting SCN juveniles had significant positive correlation with the second dimension (y-axis), they had significant correlations in opposite directions with the first dimension (x-axis) (Fig. $3 \mathrm{~B}$, vectors). None of the other nematode species explained a significant amount of variation observed in the data.

\section{DISCUSSION}

Soybean SDS is commonly observed in patches with high severity. Here, we show that many soil variables are significantly aggregated within fields, including the causal agent of SDS, $F$. virguliforme (Table 1 ). These patches of high $F$. virguliforme abundance physically overlapped with future patches of severe SDS and yield loss (Fig. 1). Because the data are spatially aggregated (Table 1), the correlations between $F$. virguliforme and SDS and yield may be driven by their geospatial location rather than their biological functions, but their overlapping distribution and etiology of $F$. virguliforme suggests a biological explanation. Other studies have also observed that higher $F$. virguliforme populations increase foliar SDS (Freed et al. 2017; Roy et al. 1997).

SDS is difficult to manage, particularly because of a lack of effective in-season strategies. Therefore, knowing the risk of SDS development ahead of time can influence decision making about purchasing partially resistant cultivars and whether or not to use a seed treatment. The present study supports previous findings that F. virguliforme inoculum has the largest contribution to SDS development and that SCN has a minor contribution to SDS development (Scherm et al. 1998). By quantifying pathogen abundance at-planting, valuable risk information can be gained and implemented into a risk prediction model for SDS development. The risk prediction models developed here show promise pending further validation in different environments. The results of crossvalidation analyses, such as the ones conducted in the present study, can be difficult to interpret (Alexander et al. 2015; Golbraikh and Tropsha 2002). All of the model $\mathrm{Q}^{2}$ values in this study were positive, indicating that the models have good potential, as poorly
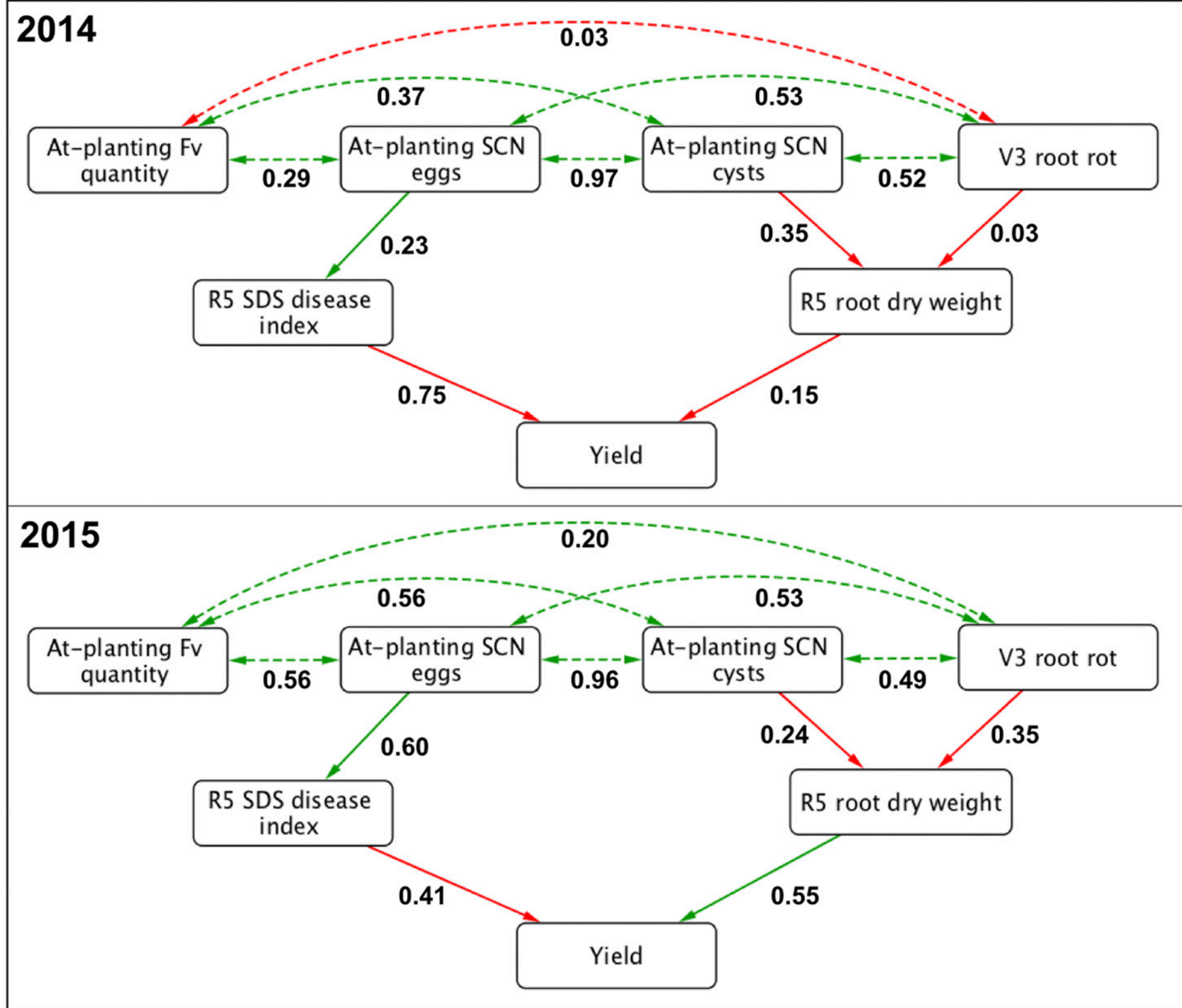

Fig. 2. Pathway analysis from at-planting risk factors to soybean growth stage R5 plant health variables, to yield in 2014 and 2015 . Numbers below arrows represent standardized parameter estimates, or beta estimates, which represent the change in standard deviations of the response variable per 1 standard deviation in the predictive variable. Green dashed lines represent positive covariate relationships, green solid lines indicate positive causal relationships, red solid lines indicate negative causal relationships. SDS $=$ sudden death syndrome, $\mathrm{Fv}=$ Fusarium virguliforme, $\mathrm{SCN}=$ soybean cyst nematode, $\mathrm{V} 3, \mathrm{R} 5=$ soybean vegetative growth stage V3 or R5, respectively. 
performing models yield negative $\mathrm{Q}^{2}$ values (Alexander et al. $2015)$. However, the $\mathrm{Q}^{2}$ values were not $>0.5$, and fail to meet the criteria for "highly accurate" models (Alexander et al. 2015; Golbraikh and Tropsha 2002). Therefore, we recommend that these models are applied cautiously and are adapted to incorporate even more data, such as soil temperature, soil moisture (Scherm and Yang 1996), photosynthesis (Bajwa et al. 2017; Kuhlgert et al. 2016), or microbiome data (Srour et al. 2017). Combining these types of biotic and environmental risk factors will likely enhance SDS risk prediction further.

$F$. virguliforme is a soilborne pathogen that infects soybean roots and remains there throughout foliar SDS development. Detailed mechanisms of foliar SDS development are complicated and largely unknown. Previous studies have shown that there is no consistent correlation between root $F$. virguliforme quantity and the amount of root rot or foliar SDS observed (Wang et al. 2019). In the present study, we also observed inconsistent correlations between root $F$. virguliforme quantities and disease symptoms. F. virguliforme quantities in V3 roots had opposite trends with R5 SDS DX in 2014 and 2015. Similarly, it had opposite trends with yield in 2014 and 2015 (Table 1). Finally, F. virguliforme quantities in R5 roots showed the exact opposite trends with R5 SDS DX and yield as $F$. virguliforme quantities in $\mathrm{V} 3$ roots (Table 1). In contrast to root qPCR quantities, the abundance of $F$. virguliforme in the soil atplanting consistently had positive correlations with R5 SDS DX and yield (Table 1). These results support the previous findings that the amount of $F$. virguliforme inside of the root does not necessarily indicate current or future disease severity (Wang et al. 2019).

Previous studies have shown that soybean vascular colonization by $F$. virguliforme is critical for severe foliar symptom development (Islam et al. 2017a, b; Navi and Yang 2008), but the F. virguliforme qPCR assay used in this study does not provide localization information when used on entire roots. The combination of high F. virguliforme quantity in the root with low foliar symptoms, or vice versa, might be explained by the location of colonization within the root. It also has been suggested that some $F$. virguliforme isolates may be good at colonizing root tissues but poor at secreting toxins, whereas others may be poor root colonizers but efficient toxin producers ( $\mathrm{Li}$ et al. 2009). Regardless, the abundance of $F$. virguliforme in the soil is valuable risk information, and pathogen quantities in postharvest soil should be examined to determine if these data are representative of pathogen quantities in the soil the following spring. If so, risk information could be obtained even earlier, allowing growers to know their risk of developing SDS before placing their cultivar and seed treatment orders.

It is well established that both SDS and SCN can cause significant yield loss in soybean independently (Hartman et al. 2015; Koenning and Wrather 2010), but many reports also have indicated a synergism between $\mathrm{SCN}$ and $F$. virguliforme that results in more severe foliar SDS in the field (Back et al. 2002; Brzostowski et al. 2014; McLean and Lawrence 1993; Scherm et al. 1998; Westphal et al. 2014; Xing and Westphal 2013). The multivariate analyses in this study indicate that the presence of both pathogens affects the severity of SDS development (Fig. 3). Interestingly, the PCoA also shows that the two pathogens have strong correlations in opposite directions along the $\mathrm{x}$-axis (Fig. 3B). This suggests that there is competition between the two pathogens for nutrients within the root, either directly or indirectly. In instances in which SCN are highly abundant, $F$. virguliforme may not be able to thrive and coexist with SCN because of competition for nutrients. Similarly, in instances in which $F$. virguliforme is highly abundant, SCN may not be able to thrive and coexist with $F$. virguliforme. Overall, more SCN juveniles were observed in 2014 than in 2015, hence it was surprising that SCN quantities had a significant correlation with R5 SDS DX and yield in 2015, not 2014. This observation could be because of the increased sampling effort and higher resolution of data collected in 2015. Taken together, our data support that $F$. virguliforme is primarily responsible for SDS development, and that the additional presence of SCN can increase the risk of developing severe SDS in a density-dependent manner.

It has been suggested that SCN or other nematodes may cause wounds and forge a path to the vascular tissue, making it easier for

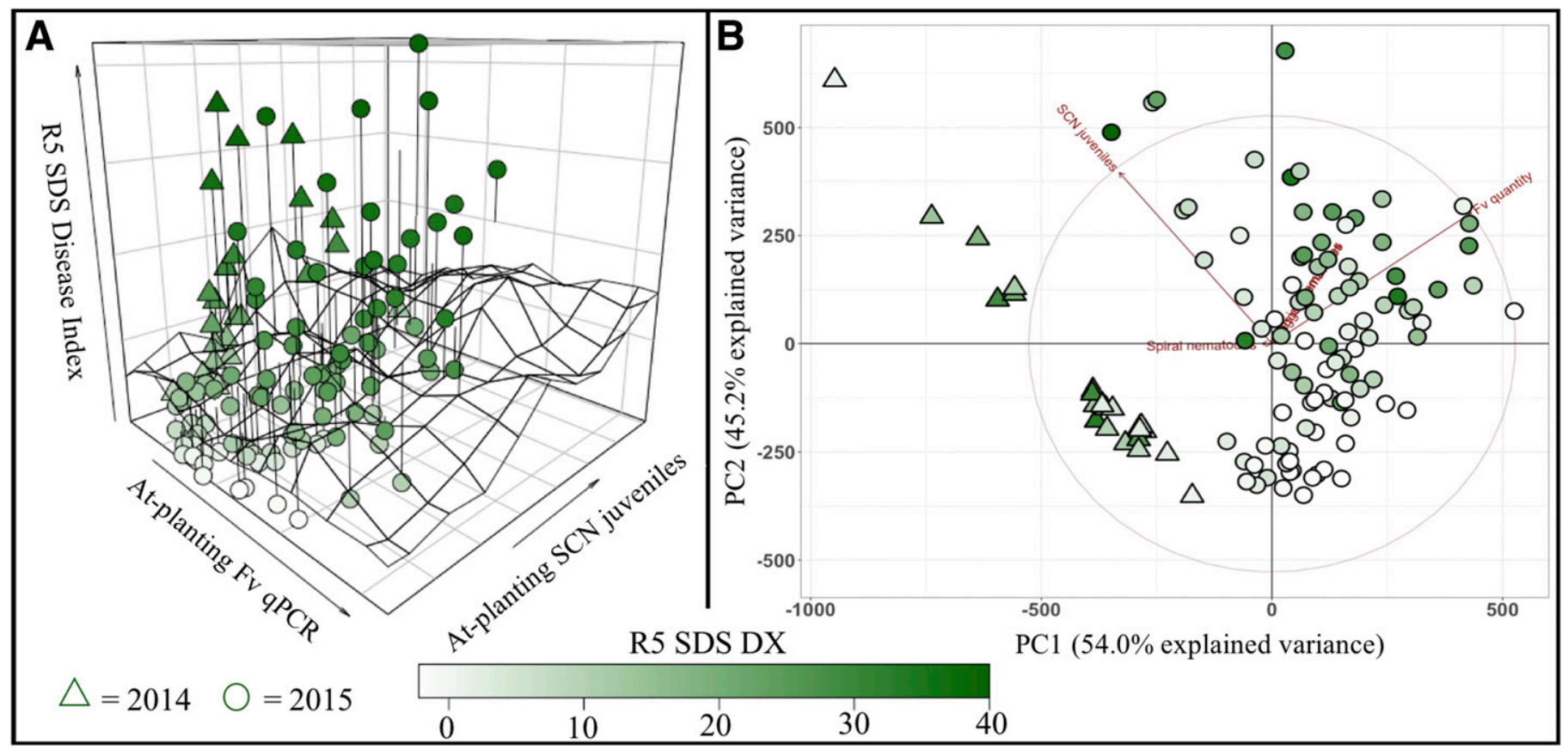

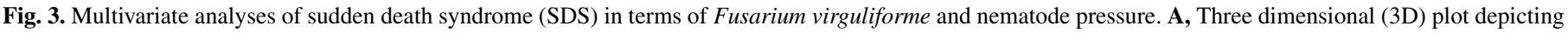

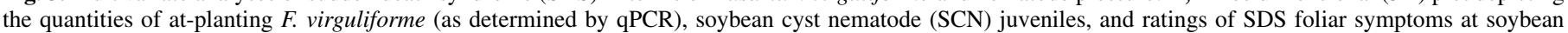

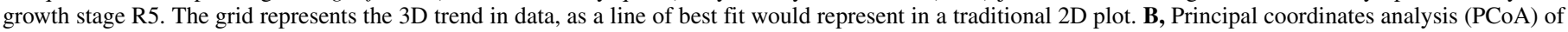

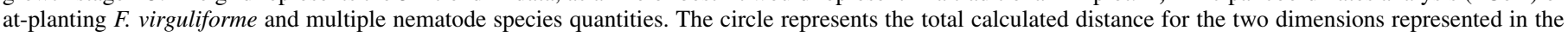

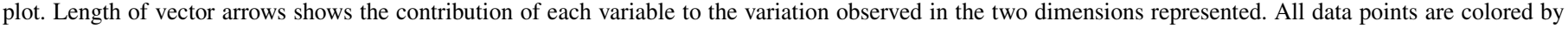
SDS disease index at stage R5. 
F. virguliforme to enter the roots (Diaz Arias 2012). Our analyses found that SCN had a significant association with SDS development, but other nematodes did not (Fig. 3B). Soybean responds to SCN infections by altering expression of pathogenesis-related genes and WRKY and MYB transcription factors (Ithal et al. 2007a). Infectious SCN juveniles also upregulate cell wall-loosening enzymes, suppress jasmonic acid (JA) in syncytia, and increase peroxidase expression to remove reactive oxygen species (Ithal et al. 2007b). These physiological responses to SCN could render soybean more susceptible to infections by $F$. virguliforme, as a reduction of JA within plants benefits necrotrophic fungi (Antico et al. 2012). It is also possible that a juvenile SCN infecting the same root as $F$. virguliforme may create an environmental change that stimulates more phytotoxin production. In fact, the pathway analysis indicates that $F$. virguliforme increases foliar SDS through interactions with $\mathrm{SCN}$ (Fig. 2). The exact mechanism by which SCN increases the amount of foliar SDS caused by $F$. virguliforme is still unknown.

Soybean yield and SDS disease severity are simple to measure, but difficult to predict because they can be affected by numerous environmental, edaphic, and biotic factors. Data from other locations should be processed through these models to further test the accuracy of each model and also determine if different models are better suited to other locations. However, the work here provides a basic framework for incorporating biotic information into risk prediction models for an economically important disease of soybean. This work is an important step toward facilitating improved SDS management and also provides insights into potential soybean yield losses that can affect soybean markets.

\section{ACKNOWLEDGMENTS}

We thank D. Kramer and D. TerAvest for helpful discussions; H.-X. Chang, J. Willbur, and J. L. Jacobs for critical review of this manuscript; J. L. Jacobs for root rot disease severity ratings; and C. Druskovich and A. Druskovich for use of fields in Decatur, MI.

\section{LITERATURE CITED}

Abeysekara, N. S., and Bhattacharyya, M. K. 2014. Analyses of the xylem sap proteomes identified candidate Fusarium virguliforme proteinaceous toxins. PLoS One 9:e93667.

Alexander, D. L. J., Tropsha, A., and Winkler, D. A. 2015. Beware of $\mathrm{R}^{2}$ : simple, unambiguous assessment of the prediction accuracy of QSAR and QSPR models. J. Chem. Inf. Model. 55:1316-1322.

Allen, T. W., Bradley, C. A., Sisson, A. J., Byamukama, E., Chilvers, M. I., Coker, C. M., Collins, A. A., Damicone, J. P., Dorrance, A. E., Dufault, N. S., Esker, P. D., Faske, T. R., Giesler, L. J., Grybauskas, A. P., Hershman, D. E., Hollier, C. A., Isakeit, T., Jardine, D. J., Kelly, H. M., Kemerait, R. C., Kleczewski, N. M., Koenning, S. R., Kurle, J. E., Malvick, D. K., Markell, S. G., Mehl, H. L., Mueller, D. S., Mueller, J. D., Mulrooney, R. P., Nelson, B. D., Newman, M. A., Osborne, L., Overstreet, C., Padgett, G. B., Phipps, P. M., Price, P. P., Sikora, E. J., Smith, D. L., Spurlock, T. N., Tande, C. A., Tenuta, A. U., Wise, K. A., and Wrather, J. A. 2017. Soybean yield loss estimates due to diseases in the United States and Ontario, Canada, from 2010 to 2014. Plant Heal. Prog. 18:19-27.

Antico, C. J., Colon, C., Banks, T., and Ramonell, K. M. 2012. Insights into the role of jasmonic acid-mediated defenses against necrotrophic and biotrophic fungal pathogens. Front. Biol. 7:48-56.

Aoki, T., O'Donnell, K., Homma, Y., and Lattanzi, A. R. 2003. Sudden-death syndrome of soybean is caused by two morphologically and phylogenetically distinct species within the Fusarium solani species complexF. virguliforme in North America and F. tucumaniae in South America. Mycologia 95:660-684.

Back, M. A., Haydock, P. P. J., and Jenkinson, P. 2002. Disease complexes involving plant parasitic nematodes and soilborne pathogens. Plant Pathol. 51:683-697.

Bajwa, S. G., Rupe, J. C., and Mason, J. 2017. Soybean disease monitoring with leaf reflectance. Remote Sens. 9:127.

Bez, N. 2000. On the use of Lloyd's index of patchiness. Fish. Oceanogr. 9: 372-376.

Brar, H. K., Swaminathan, S., and Bhattacharyya, M. K. 2011. The Fusarium virguliforme toxin FvTox1 causes foliar sudden death syndrome-like symptoms in soybean. Mol. Plant-Microbe Interact. 24:1179-1188.
Bray, R. J., and Curtis J. T. 1957. An ordination of the upland forest communities of southern Wisconsin. Ecol. Monogr. 27:325-349.

Brzostowski, L. F., Schapaugh, W. T., Rzodkiewicz, P. A., Todd, T. C., and Little, C. R. 2014. Effect of host resistance to Fusarium virguliforme and Heterodera glycines on sudden death syndrome disease severity and soybean yield. Plant Heal. Prog. 15:1-8.

Chang, H.-X., Roth, M. G., Wang, D., Cianzio, S. R., Lightfoot, D. A., Hartman, G. L., and Chilvers, M. I. 2018. Integration of sudden death syndrome resistance loci in the soybean genome. Theor. Appl. Genet. 131: 757-773.

Chang, H.-X., Yendrek, C. R., Caetano-Anolles, G., and Hartman, G. L. 2016. Genomic characterization of plant cell wall degrading enzymes and in silico analysis of xylanases and polygalacturonases of Fusarium virguliforme. BMC Microbiol. 16:147.

de Farias Neto, A. L., Hashmi, R., Schmidt, M., Carlson, S. R., Hartman, G. L., Li, S., Nelson, R. L., and Diers, B. W. 2007. Mapping and confirmation of a new sudden death syndrome resistance QTL on linkage group D2 from the soybean genotypes PI 567374 and "Ripley". Mol. Breed. 20: 53-62.

Del Ponte, E. M., Godoy, C. V., Li, X., and Yang, X. B. 2006. Predicting severity of Asian soybean rust epidemics with empirical rainfall models. Phytopathology 96:797-803.

Diaz Arias, M. M. 2012. Fusarium species infecting soybean roots: Frequency, aggressiveness, yield impact and interaction with the soybean cyst nematode. Graduate Theses and Dissertations. Iowa State University, Paper 12314.

Donald, P. A., Niblack, T. L., and Wrather, J. A. 1993. First report of Fusarium solani blue isolate, a causal agent of sudden death syndrome of soybeans, recovered from soybean cyst nematode eggs. Plant Dis. 77:647.

Epskamp, S. 2017. semPlot: Path diagrams and visual analysis of various SEM packages' output. https://cran.r-project.org/web/packages/semPlot/index.html

Faith, D. P., Minchin, P. R., and Belbin, L. 1987. Compositional dissimilarity as a robust measure of ecological distance. Vegetatio 69:57-68.

Fehr, W. R., Caviness, C. E., Burmood, D. T., and Pennington, J.S. 1971. Stage of development description for soybeans, Glycine max (L.). Merrill. Crop Sci. 11:929-931.

Freed, G. M., Floyd, C. M., and Malvick, D. K. 2017. Effects of pathogen population levels and crop-derived nutrients on development of soybean sudden death syndrome and growth of Fusarium virguliforme. Plant Dis. $101: 434-441$

Gao, X., Jackson, T. A., Hartman, G. L., and Niblack, T. L. 2006. Interactions between the soybean cyst nematode and Fusarium solani f. sp. glycines based on greenhouse factorial experiments. Phytopathology 96:1409-1415.

Gaspar, A. P., Mueller, D. S., Wise, K. A., Chilvers, M. I., Tenuta, A. U., and Conley, S. P. 2017. Response of broad-spectrum and target-specific seed treatments and seeding rate on soybean seed yield, profitability, and economic risk. Crop Sci. 57:2251-2262.

Golbraikh, A., and Tropsha, A. 2002. Beware of q2! J. Mol. Graph. Model. 20: 269-276.

Gongora-Canul, C. C., and Leandro, L. F. S. 2011. Effect of soil temperature and plant age at time of inoculation on progress of root rot and foliar symptoms of soybean sudden death syndrome. Plant Dis. 95:436-440.

Hartman, G. L., Chang, H.-X., and Leandro, L. F. 2015. Research advances and management of soybean sudden death syndrome. Crop Prot. 73:60-66.

Hershman, D. E., Hendrix, J. W., Stuckey, R. E., and Bachi, P. R. 1990. Influence of planting date and cultivar on soybean sudden death syndrome in Kentucky. Plant Dis. 74:761-766.

Hughes, T. J., O'Donnell, K., Sink, S., Rooney, A. P., Scandiani, M. M., Luque, A., Bhattacharyya, M. K., and Huang, X. 2017. Genetic architecture and evolution of the mating type locus in fusaria that cause soybean sudden death syndrome and bean root rot. Mycologia 106: 686-697.

Islam, K. T., Bond, J. P., and Fakhoury, A. M. 2017a. FvSNF1, the sucrose non-fermenting protein kinase gene of Fusarium virguliforme, is required for cell-wall-degrading enzymes expression and sudden death syndrome development in soybean. Curr. Genet. 63:723-738.

Islam, K. T., Bond, J. P., and Fakhoury, A. M. 2017b. FvSTR1, a striatin orthologue in Fusarium virguliforme is required for asexual development and virulence. Appl. Microbiol. Biotechnol. 101:6431-6445.

Ithal, N., Recknor, J., Nettleton, D., Hearne, L., Maier, T., Baum, T. J., and Mitchum, M. G. 2007a. Parallel genome-wide expression profiling of host and pathogen during soybean cyst nematode infection of soybean. Mol. Plant-Microbe Interact. 20:293-305.

Ithal, N., Recknor, J., Nettleton, D., Maier, T., Baum, T. J., and Mitchum, M. G. 2007b. Developmental transcript profiling of cyst nematode feeding cells in soybean roots. Mol. Plant-Microbe Interact. 20:510-525.

Jenkins, W. R. 1964. A rapid centrifugal-flotation technique for separating nematodes from soil. Plant Dis. Rep. 48:692. 
Kandel, Y. R., Mueller, D. S., Legleiter, T., Johnson, W. G., Young, B. G., and Wise, K. A. 2018. Impact of fluopyram fungicide and preemergence herbicides on soybean injury, population, sudden death syndrome, and yield. Crop Prot. 106:103-109.

Kandel, Y. R., Wise, K. A., Bradley, C., Chilvers, M. I., Byrne, A. M., Tenuta, A., Faghihi, J., Wiggs, S. N., and Mueller, D. S. 2017. Effect of soybean cyst nematode resistance source and seed treatment on population densities of Heterodera glycines, sudden death syndrome, and yield of soybean. Plant Dis. 101:2137-2143.

Kandel, Y. R., Wise, K. A., Bradley, C. A., Chilvers, M. I., Tenuta, A. U., and Mueller, D. S. 2016. Fungicide and cultivar effects on sudden death syndrome and yield of soybean. Plant Dis. 100:1339-1350.

Kazi, S., Shultz, J., Afzal, J., Johnson, J., Njiti, V. N., and Lightfoot, D. A. 2008. Separate loci underlie resistance to root infection and leaf scorch during soybean sudden death syndrome. Theor. Appl. Genet. 116: 967-977.

Koenning, S. R., and Wrather, J. A. 2010. Suppression of soybean yield potential in the continental United States by plant diseases from 2006 to 2009. Plant Heal. Prog. doi:10.1094/PHP-2010-1122-01-RS

Kolander, T. M., Bienapfl, J. C., Kurtle, J. E., and Malvick, D. K. 2012. Symptomatic and asymptomatic host range of Fusarium virguliforme, the causal agent of soybean sudden death syndrome. Plant Dis. 96: 1148-1153.

Kuhlgert, S., Austic, G., Zegarac, R., Osei-Bonsu, I., Hoh, D., Chilvers, M. I., Roth, M. G., Bi, K., TerAvest, D., Weebadde, P., and Kramer, D. M. 2016. MultispeQ Beta: a tool for large-scale plant phenotyping connected to the open PhotosynQ network. R. Soc. Open Sci. 3:160592.

Kuhn, M. 2018. caret: Classification and regression training. https://cran.rproject.org/web/packages/caret/index.html

Kutner, M. H., Nachtsheim, C. J., Neter, J., and Li, W. 2005. Multicollinearity and its effects. Pages 278-293 in: Applied Linear Statistical Models, 5th ed. McGraw Hill, Boston.

Leandro, L. F. S., Eggenberger, S., Chen, C., Williams, J., Beattie, G. A., and Liebman, M. 2018. Cropping system diversification reduces severity and incidence of soybean sudden death syndrome caused by Fusarium virguliforme. Plant Dis. 102:1748-1758.

Li, S., Hartman, G. L., and Chen, Y. 2009. Evaluation of aggressiveness of Fusarium virguliforme isolates that cause soybean sudden death syndrome. J. Plant Pathol. 91:77-86.

Lightfoot, D. A. 2015. Two decades of molecular marker-assisted breeding for resistance to soybean sudden death syndrome. Crop Sci. 55:1460-1484.

Lloyd, M. 1967. Mean crowding. J. Anim. Ecol. 36:1-30.

Luckew, A. S., Leandro, L. F., Bhattacharyya, M. K., Nordman, D. J., Lightfoot, D. A., and Cianzio, S. R. 2013. Usefulness of 10 genomic regions in soybean associated with sudden death syndrome resistance. Theor. Appl. Genet. 126:2391-2403.

Luo, Y., Hildebrand, K., Chong, S. K., Myers, O., and Russin, J. S. 2000. Soybean yield loss to sudden death syndrome in relation to symptom expression and root colonization by Fusarium solani f. sp. glycines. Plant Dis. 84:914-920.

Marburger, D., Conley, S., Esker, P., MacGuidwin, A., and Smith, D. 2014. Relationship between Fusarium virguliforme and Heterodera glycines in commercial soybean fields in Wisconsin. Plant Heal. Prog. 15:11-18.

McLean, K. S., and Lawrence, G. W. 1993. Interrelationship of Heterodera glycines and Fusarium solani in sudden death syndrome of soybean. J. Nematol. 25:434-439.

McLean, K. S., and Lawrence, G. W. 1995. Development of Heterodera glycines as affected by Fusarium solani, the causal agent of sudden death syndrome of soybean. J. Nematol. 27:70-77.

Meksem, K., Doubler, T. W., Chancharoenchai, K., Njiti, V. N., Chang, S. J. C., Rao Arelli, A. P., Cregan, P. E., Gray, L. E., Gibson, P. T., and Lightfoot, D. A. 1999. Clustering among loci underlying soybean resistance to Fusarium solani, SDS and SCN in near-isogenic lines. Theor. Appl. Genet. 99:1131-1142.

Mila, A. L., Carriquiry, A. L., and Yang, X. B. 2004. Logistic regression modeling of prevalence of soybean Sclerotinia stem rot in the north-central region of the United States. Phytopathology 94:102-110.

Navi, S. S., and Yang, X. B. 2008. Foliar symptom expression in association with early infection and xylem colonization by Fusarium virguliforme (formerly $F$. solani f. sp. glycines), the causal agent of soybean sudden death syndrome. Plant Heal. Prog. doi:10.1094/PHP-2008-0222-01-RS

Navi, S. S., and Yang, X. B. 2016. Impact of crop residue and corn-soybean rotation on the survival of Fusarium virguliforme a causal agent of sudden death syndrome of soybean. J. Plant Pathol. Microbiol. 7:1-7.

Njiti, V. N., Johnsona, J. E., Torto, T. A., Gray, L. E., and Lightfoot, D. A. 2001. Inoculum rate influences selection for field resistance to soybean sudden death syndrome in the greenhouse. Crop Sci. 41:1726-1731.

Noble, W. S. 2009. How does multiple testing correction work? Nat. Biotechnol. 27:1135-1137.
Nychka, D., Furrer, R., Paige, J., and Sain, S. 2017. fields: Tools for spatial data. doi:10.5065/D6W957CT

O'Brien, R. M. 2007. A caution regarding rules of thumb for variance inflation factors. Qual. Quant. 41:673-690.

Oksanen, J., Blanchet, F. G., Friendly, M., Kindt, R., Legendre, P., McGlinn, D., Minchin, P. R., O’Hara, R. B., Simpson, G. L., Solymos, P., Stevens, M. H. H., Szoecs, E., and Wagner, H. 2018. vegan: Community ecology package. https://cran.r-project.org/web/packages/vegan/index.html

Pudake, R. N., Swaminathan, S., Sahu, B. B., Leandro, L. F., and Bhattacharyya, M. K. 2013. Investigation of the Fusarium virguliforme fvtox 1 mutants revealed that the FvTox 1 toxin is involved in foliar sudden death syndrome development in soybean. Curr. Genet. 59:107-117.

Rosseel, Y. 2012. lavaan: An R package for structural equation modeling. J. Stat. Softw. 48:1-36.

Rossman, D. R., Byrne, A. M., and Chilvers, M. I. 2018. Profitability and efficacy of soybean seed treatment in Michigan. Crop Prot. 114: 44-52.

Roy, K. W., Hershman, D. E., Rupe, J. C., and Abney, T. S. 1997. Sudden death syndrome of soybean. Plant Dis. 81:1100-1111.

Roy, K. W., Lawrence, G. W., Hodges, H. H., McLean, K. S., and Killebrew, J. F. 1989. Sudden death syndrome of soybean: Fusarium solani as incitant and relation of Heterodera glycines to disease severity. Phytopathology 79:191-197.

Rupe, J. C. 1989. Frequency and pathogenicity of Fusarium solani recovered from soybeans with sudden death syndrome. Plant Dis. 73:581-584.

Rupe, J. C., Sabbe, W. E., Robbins, R. T., and Gbur, E. E. 1993. Soil and plant factors associated with sudden-death syndrome of soybean. J. Prod. Agric. $6: 218-221$

Sang, H., Witte, A., Jacobs, J. L., Chang, H.-X., Wang, J., Roth, M. G., and Chilvers, M. I. 2018. Fluopyram sensitivity and functional characterization of SdhB in the Fusarium solani species complex causing soybean sudden death syndrome. Front. Microbiol. 9:2335.

Scherm, H., and Yang, X. B. 1996. Development of sudden death syndrome of soybean in relation to soil temperature and soil water matric potential. Phytopathology 86:642-649.

Scherm, H., and Yang, X. B. 1999. Risk assessment for sudden death syndrome of soybean in the north-central United States. Agric. Syst. 59: 301-310.

Scherm, H., Yang, X. B., and Lundeen, P. 1998. Soil variables associated with sudden death syndrome in soybean fields in Iowa. Plant Dis. 82:1152-1157.

Shannon, P., Markiel, A., Ozier, O., Baliga, N. S., Wang, J. T., Ramage, D., Amin, N., Schwikowski, B., and Ideker, T. 2003. Cytoscape: A software environment for integrated models of biomolecular interaction networks. Genome Res. 13:2498-2504.

Soetaert, K. 2017. plot3D: Plotting multi-dimensional data. https://cran.rproject.org/web/packages/plot3D/index.html

Srour, A., Afzal, A. J., Blahut-Beatty, L., Hemmati, N., Simmonds, D. H., Li, W., Liu, M., Town, C. D., Sharma, H., Arelli, P., and Lightfoot, D. A. 2012. The receptor like kinase at $R h g 1-a / R f s 2$ caused pleiotropic resistance to sudden death syndrome and soybean cyst nematode as a transgene by altering signaling responses. BMC Genomics 13:368.

Srour, A. Y., Gibson, D. J., Leandro, L. F. S., Malvick, D. K., Bond, J. P., and Fakhoury, A. M. 2017. Unraveling microbial and edaphic factors affecting the development of sudden death syndrome in soybean. Phytobiomes J. 1: 91-101.

Tan, R., Collins, P. J., Wang, J., Wen, Z., Boyse, J. F., Laurenz, R. G., Gu, C., Jacobs, J. L., Song, Q., Chilvers, M. I., and Wang, D. 2019. Different loci associated with root and foliar resistance to sudden death syndrome (Fusarium virguliforme) in soybean. Theor. Appl. Genet. 132:501-513.

Venables, W. N., and Ripley, B. D. 2002. Modern Applied Statistics with S, 4th ed. Springer, New York.

Vu, V. Q. 2011. ggbiplot: A ggplot2 based biplot. https://github.com/vqv/ ggbiplot

Wang, J., Bradley, C., Stenzel, O., Pedersen, D. K., Reuter-Carlson, U., and Chilvers, M. I. 2016. Baseline sensitivity of Fusarium virguliforme to fluopyram fungicide. Plant Dis. 101:576-582.

Wang, J., Jacobs, J. L., Byrne, J. M., and Chilvers, M. I. 2015. Improved diagnoses and quantification of Fusarium virguliforme, causal agent of soybean sudden death syndrome. Phytopathology 105:378-387.

Wang, J., Jacobs, J. L., Roth, M. G., and Chilvers, M. I. 2019. Temporal dynamics of Fusarium virguliforme colonization of soybean roots. Plant Dis. 103:19-27.

Weems, J. D., Haudenshield, J. S., Bond, J. P., Hartman, G. L., Ames, K. A., and Bradley, C. A. 2015. Effect of fungicide seed treatments on Fusarium virguliforme infection of soybean and development of sudden death syndrome. Can. J. Plant Pathol. 37:435-447.

Wen, Z., Tan, R., Yuan, J., Bales, C., Du, W., Zhang, S., Chilvers, M. I., Schmidt, C., Song, Q., Cregan, P. B., and Wang, D. 2014. Genome-wide association mapping of quantitative resistance to sudden death syndrome in soybean. BMC Genomics 15:809. 
Westphal, A., Li, C., Xing, L., McKay, A., and Malvick, D. 2014. Contributions of Fusarium virguliforme and Heterodera glycines to the disease complex of sudden death syndrome of soybean. PLoS One 9:e99529.

Willbur, J. F., Fall, M. L., Bloomingdale, C., Byrne, A. M., Chapman, S. A., Magarey, R. D., McCaghe, M. M., Bueller, B. D., Russo, J. M., Schlegel, J., Chilvers, M. I., Mueller, D. S., Kabbage, M., and Smith, D. L. 2018a. Weather-based models for assessing the risk of Sclerotinia sclerotiorum apothecial presence in soybean (Glycine max) fields. Plant Dis. 102: 73-84
Willbur, J. F., Fall, M. L., Byrne, A. M., Chapman, S. A., McCaghey, M. M., Mueller, B. D., Schmidt, R., Chilvers, M. I., Mueller, D. S., Kabbage, M., Giesler, L. J., Conley, S. P., and Smith, D. L. 2018 b. Validating Sclerotinia sclerotiorum apothecial models to predict Sclerotinia stem rot in soybean (Glycine max) fields. Plant Dis. 102: 2592-2601.

Xing, L., and Westphal, A. 2013. Synergism in the interaction of Fusarium virguliforme with Heterodera glycines in sudden death syndrome of soybean. J. Plant Dis. Prot. 120:209-217. 